\title{
Dank an die Gutachter
}

Wir danken allen Gutachterinnen und Gutachtern herzlich, die durch ihre Unterstützung im vergangenen Jahr maßgeblich zur Qualität von VeRHALTENSTHERAPIE beigetragen haben, namentlich:

Dr. Judith Alder, Basel PD Dr. Georg Alpers, Würzburg PD Dr. Matthias Backenstraß, Heidelberg

Dr. Thomas Bär, Rostock

Prof. Dr. Matthias Berking, Bern Prof. Dr. Martin Bohus, Mannheim Prof. Dr. Gerhard Bühringer, Dresden PD Dr. Cornelia Exner, Marburg Prof. Dr. Manfred Fichter,

Prien am Chiemsee

Dr. Guido Flatten, Aachen

Prof. Dr. Harald J. Freyberger, Greifswald

PD Dr. Alexander L. Gerlach, Münster Prof. Dr. Iver Hand, Hamburg Prof. Dr. Thomas Heidenreich, Esslingen/N.

Prof. Dr. Nina Heinrichs, Bielefeld PD Dr. Anja Hilbert, Marburg Dr. Eva Hoch, Dresden Prof. Dr. Jürgen Hoyer, Dresden PD Dr. Klaus Junghanns, Lübeck Prof. Dr. Kerstin Konrad, Aachen Dr. Andreas Kordon, Lübeck
Prof. Dr. Birgit Kröner-Herwig, Göttingen

Dr. Tanja Legenbauer, Dortmund Prof. Dr. Fritz Mattejat, Marburg Prof. Dr. Rolf Meermann, Bad Pyrmont PD Dr. Tanja Michael, Basel Prof. Dr. Frank Neuner, Bielefeld Dr. Jörg Petry, Neunkirchen Prof. Dr. Babette Renneberg, Berlin Prof. Dr. Hertha Richter-Appelt, Hamburg

Prof. Dr. Dieter Riemann, Freiburg i.Br. Prof. Dr. Fred Rist, Münster

Dr. Maggie Schauer,

Reichenau-Lindenbühl

PD Dr. Elisabeth Schramm, Freiburg i.Br.

Prof. Dr. Ulrich Schweiger, Lübeck

Prof. Dr. Rolf-Dieter Stieglitz, Basel Prof. Dr. Dr. Harald Walach,

Northampton

Prof. Dr. Michael Zaudig,

Windach/Ammersee

Prof. Dr. Dirk Zimmer, Tübingen

Die Herausgeber von Verhaltenstherapie 2008

Prof. Dr. Ulrike Ehlert, Zürich Prof. Dr. Manfred M. Fichter, Prien am Chiemsee Prof. Dr. Fritz. Hohagen, Lübeck Prof. Dr. Michael Linden, Berlin Prof. Dr. Winfried Rief, Marburg 